\title{
Hydroxy Propyl Methyl Cellulose: Different Aspects in
}

\section{Drug Delivery}

\author{
Tamasree Majumder, Gopa Roy Biswas and Sutapa Biswas Majee \\ Department of Pharmaceutical Technology, NSHM Knowledge Campus-Group of Institutions, Kolkata 700140, West Bengal, India
}

\begin{abstract}
Among the various research works going on nowadays, designing of controlled release dosage form is of great importance. For the development of suitable controlled release dosage form, a proper matrix needs to be formed from which the drug release generally occur by polymer swelling, polymer erosion, drug dissolution/diffusion mechanism. HPMC (hydroxy propyl methyl cellulose), also known as hypromellose, is one of the best known cellulosic polymers used in the development of controlled released drug delivery. It is available in various grades. Cellulosic polymers are ingredients that contain units linked together which help to retain water. Due to its high water absorptive capacity, it acts as an excellent hydrophilic gel forming polymer. HPMC generally hydrates on the outer surface to form a gelatinous layer which is critical to prevent wetting and rapid drug release from the matrices. If the drug is sparingly soluble in the system, the release of drug from the system is slow and helps in formulation of controlled release dosage form. In the ophthalmic dosage form, HPMC is used as a matrix that swells and expands after absorbing water and expand the thickness of the tear film.
\end{abstract}

Key words: HPMC, hypromellose, hydrophilic, controlled released dosage form.

\section{Introduction}

HPMC (hydroxy propyl methyl cellulose) is a partly o-methylated and o-(2-hydroxyl propylated) cellulose, obtained by treating alkali cellulose with chloromethane and propylene oxide. It is odorless and tasteless granular or fibrous powder which is creamy white or white in color. HPMC is soluble in cold water. Aqueous dispersions are prepared by dispersing material in about $25 \%$ hot water $\left(80{ }^{\circ} \mathrm{C}\right)$ under vigorous stirring. On complete hydration of HPMC, a sufficient quantity of cold water is added and mixed. The gel point of HPMC dispersions varies from $50{ }^{\circ} \mathrm{C}$ to $90{ }^{\circ} \mathrm{C}$. Gels are stable over a wide $\mathrm{pH}$ range (3-1). The viscosity of HPMC dispersions depends on the concentration of material used, its molecular weight, vehicle composition, presence of preservatives, and so on. Viscous gels can be prepared using high concentrations of high-molecular-weight grades. Inclusion of organic solvents, such as ethanol or

Corresponding author: Tamasree Majumder, post-graduate fellow, research fields: controlled drug delivery, preformulation and dosage form design. viscosity, can be altered upon the blending of the components. A blending formula is available which is a very useful tool for determine the viscosity of the final HPMC preparation that we want to produce. The viscosity of a blend can, however, be approximated by use of the equation below, which is derived from the Arrhenius equation that relates viscosity with polymer concentration.

$$
\log V_{s}=\left\{n \log V_{1}+(100-n) \log V_{2}\right\} / 100
$$

where:

$V_{s}=$ viscosity sought;

$n=$ percent (by weight) of the first component of the blend having a viscosity of $V_{1}$;

$V_{2}=$ viscosity of the second component of the blend;

It should be noted that all viscosities must be expressed at the same polymer concentration and in the same units.

Dichloromethane improves the viscosity.

Addition of an antimicrobial preservative (e.g., benzalkonium chloride) minimizes microbial spoilage of HPMC gels [1]. HPMC is a semi-synthetic, 
viscoelastic, non-ionic hydrophilic polymer which is used extensively in various formulations of controlled release dosage form, as well as in ophthalmic preparations as lubricants. HPMC exhibits $\mathrm{pH}$ independent drug release profile [2]. Extensive use of HPMC in controlled drug delivery system has been realized because they offer unique properties so far not been attained by any other polymers. The rate of the drug release from a matrix product depends on the relaxation of the polymer chains, which overall displays sustained release characteristics [3].

HPMC produces an elastic and tough coating which is highly consistent, economical, printable, non-allergenic, non-calorigenic, micro structural and are more resistant to microbial attack. With the use of HPMC not only controlled released dosage form are prepared but tablets with minimum efficacy (drugs showing tailored drug release profile) and optimum speed can be manufactured [4]. The research about using HPMC in the controlled released dosage form is going on for about four decades. This is because, when HPMC is used as a polymeric matrix for the controlled released dosage form, the release of the drug takes place at a steady state rather than the controlled released dosage form which has been coated with normal coating ingredient. This results in uniform drug release with very lower chances of adverse effects like dose dumping [5].

\section{Function of HPMC in Controlled Releases Dosage Form}

HPMC has various uses in the field of controlled released dosage form. The function abilities are as follows:

- viscolizing agent, i.e., thickening agent;

- as coating polymer in controlled released dosage form;

- bio adhesives;

- in solid dispersion to enhance drug solubility;
- as binder in controlled release dosage form;

- coating agent;

- suspending agent;

- viscosity increasing agent.

\section{Benefits of Using HPMC in the Controlled Release Dosage Form}

There are various reasons due to which HPMC is used widely.

\subsection{Consumer Acceptance}

With the proper use of plasticizers, application rates, polymer concentration and application method excellent finishing of the product can be achieved. They can form a clear, sharp coatings and compatible with various dyes, colors and pigments that are approved by the FD\&C. Hence consumer acceptance of tablets coated with HPMC increases. In case of HPMC, a clear cut of quality effective image can be obtained which shows that the tablets coated with HPMC easy swells up in the target site and shows desired mechanism of action.

\subsection{Versatility}

HPMC is versatile in nature because it has a wide range of viscosities, grades and classes (Fig. 1). According to the type of the product that is required, the viscosity grades can be substituted with each other to get the desired coating solution. The coating solution can be prepared in such a way that the solution can be optimized keeping other parameters constant.

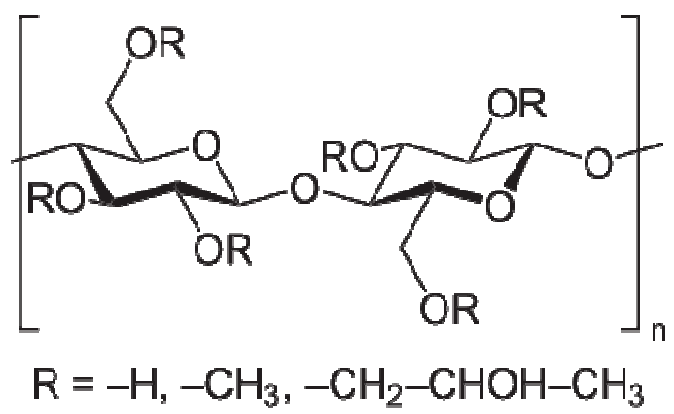

Fig. 1 Structure of HPMC. 


\section{Physical Properties of Tablet}

HPMC is compatible with a variety of ingredients that are used in the preparation of tablets. It helps in maintaining the shelf life of the product because by the virtue of its property it prevents microbial contamination and hence the degradation of the product. It can reduce tablet friability by increasing the strength of the coating [6].

\section{Drug Release}

When HPMC is used as a coating polymer the release of the drug takes place at a steady state, i.e., zero order rate kinetics is followed. Hence a constant rate of drug release is obtained and hence this increases the patient compliance.

\section{Use of HPMC in Different Drug Delivery Systems}

\subsection{Systems with Eroding or Soluble Barrier Coatings}

HPMC can be well used in the preparations of drug delivery systems with eroding or soluble barrier coatings. These types of delivery systems contain reservoir devices which are coated with a barrier layer. The barrier dissolves or erodes after a specified lag period, so that the drug can release rapidly from the reservoir core. The lag time prior to drug release from a reservoir-type device can be controlled by the thickness of the coating layer. This chronotropic systems consists of a drug-containing core layered with HPMC, optionally coated with an outer enteric coating. The lag time prior to drug release can be further controlled by the thickness and the viscosity grade of the HPMC layer. After erosion or dissolution of the HPMC layer, a distinct pulse is observed [7]. This system probably works better for poorly water soluble drug because highly water soluble drugs could diffuse through the swollen HPMC layer prior to complete erosion.

\subsection{Systems with Rupturable Coatings}

HPMC can be used as swelling agent in this type of preparation and thus results in rupture. Water influx is through the semipermeable rupturable outer coating which leads to expansion and erosion of an intermediate layer, which ultimately resulted in rupture of the outer coating the drug was then released rapidly within a short period after certain lag time due to the strong rupturing of the coating [8].

\subsection{Systems with Prolonged Contact Time}

In order to get prolong precorneal contact time and subsequently improve ocular bioavailability of the drug. HPMC offers prolonged contact time for the formulation when it is used in the ophthalmic preparation as a viscosity-enhancing agent [9].

\section{Coating Spray Solution Formulation of HPMC}

The HPMC alone is not used for the development of the polymer matrix and gelation. Along with HPMC, specific solvent is also required for the coating spray matrix development. For the formation of the coating solution, $10 \sim 15 \%$ concentrations are made when the HPMC is of low viscosity and 2 3\% concentration is taken when high viscosity HPMC is of higher viscosity [10].

\subsection{Classes of HPMC According to Uses}

HPMC is a non-ionic polymer where various viscosity grades, such as HPMC K4M, HPMC K15M and HPMC K100M, are used.

HPMC is a non-ionic polymer and hence the matrices exhibits $\mathrm{pH}$ independent drug release profiles when drug solubility is $\mathrm{pH}$ independent. However, when drug is acidic or basic drugs, the release profile may be affected by the $\mathrm{pH}$ of the media. In some cases, a $\mathrm{pH}$ independent performance in the gastrointestinal tract can lead to the consistent bioavailability of the drug [11].

\subsection{Different Grades of HPMC Based on Substitution}

HPMC is a polymeric substance which is used in 
Table 1 Showing uses of HPMC in various formulations.

\begin{tabular}{llll}
\hline No. & Location & Example & Viscosity grades \\
\hline 1 & Gastro retentive & Lisinopril, famotidine & HPMC K15M, HPMC K100M \\
2 & Buccoadhesives & Sumatriptan & HPMC:LER $=1: 1$ \\
3 & Mucoadhesives & Chlorhexidine & HPMC K4MCR \\
\hline
\end{tabular}

Table 2 Various grades of HPMC depending on substitution of methoxy and hydroxy propyl group.

\begin{tabular}{lllll}
\hline Group & HPMC grades & Viscosity $(\mathrm{Cps})$ & Methoxy $(\%)+$ hydroxy propyl (\%) & Remark \\
\hline \multirow{4}{*}{ Group-1 } & E3-LV & 3 & $29.2+8.4$ & \\
& E3-LV* & 5 & $29.1+7.7$ & Low viscosity and medium \\
& E6-LV & 6 & $29.1+8.3$ & substitution ratio grade \\
& E15-LV & 15 & $29.1+9.4$ & \\
\hline Group-2 & E50-LV* & 50 & $29.0+8.5$ & Low viscosity and low \\
\hline Group-3 & K100LV* & 100 & $22.8+9.6$ & Substitution ratio grade \\
\hline \multirow{5}{*}{ Group-4 } & K15M & 4,000 & $22.9+8.3$ & High viscosity and low \\
& K100M* & 15,000 & $22.8+8.8$ & Substitution \\
& E4M* & 100,000 & $23.3+10.8$ & High viscosity and medium \\
\hline
\end{tabular}

the preparation of the controlled released drugs but their composition varies depending on their substitution of the methyl and hydroxy propyl groups present in their structure (Tables 1 and 2).

\subsection{Effects of Various Grades of HPMC on Drug Release}

For the preparation of controlled released dosage form various grades of HPMC, such as Methocel E5, E50, K4M and K100. The various types of grades of HPMC provide log phases of varying duration and proper quantitative models of controlled released dosage form.

\section{Approximate Gel Points of HPMC Products}

Two percent aqueous solution: Gel points play a very important role in the development of matrix. This depends largely on the grades of HPMC that are used.

\section{Mechanism of Action of HPMC}

Drug release from HPMC matrices follows the classical Higuchi dissolution equation, related drug release with square root of time. Swell able systems consisting of hydrophilic polymers in the presence of water absorb a significant amount of water to form a gel. As dissolution medium penetrate the matrix, polymer material swelling starts and drug molecules begin to move out of the system by diffusion [12].

\section{Various Kinetics Model of Drug Release by HPMC}

Controlled released dosage form follows various mechanism of drug release. Hence various rate kinetics models are also developed to explain their drug release profile. The model dependent approach includes zero order, first order, Higuchi model, Hixon Crowell and Korsemeyer Peppas model [13].

In case of controlled released dosage form, when HPMC is used as a polymer, the drug is released at a constant rate so that constant dose of drug is maintained and a steady state is reached in due course of time [14].

\section{Conclusions}

HPMC is used as coating polymer, as well as matrix former in various drug deliveries. Due to its gel forming nature, it can be well accepted in controlled release drug delivery. The addition of ionic polymers to HPMC increases its drug release profile. It is light in weight hence it can be easily used to form gastro 
retentive drug delivery specially in floating drug delivery. Hence it can be stated that it has versatile uses in drug delivery.

\section{References}

[1] Doelker, E. 1993. "Cellulose Derivatives." Adv. Polym. Sci. 107: 199-265.

[2] Rell, M. T., Tiwari, B. S., and Krayz, T. G. 2009. "Formulating Hydrophilic Matrix System." Drug Deliv. Tech. 9: 20-4.

[3] Williams, H. D., Ward, R., Hardy, I. J., and Melia, C. D. 2011. "Drug Release from HPMC Matrices in Milk and Fat-Rich Emulsion." Journal of Pharmaceutical Sciences 100 (11): 4823-35.

[4] O'Neil, J. 2010. “Controlled Drug Delivery for the Application of Extended or Sustained Release Drug Products for Parenteral Administration.” M.Sc. thesis, Department of Chemistry and Chemical Biology, Northern University, 15-26.

[5] Zajic, L., and Buckton, G. 1990. "The Use of Surface Energy Values to Predict Optimum Binder Selection for Granulation.” Int. J. Pharm. 59 (2): 155-64.

[6] Sangalli, M. E., Maroni, A., Foppoli, A., Zema, L., Giordano, F., and Gazzaniga, A. 2004. "Different HPMC Viscosity Grades as Coating Agents for an Oral Time and/or Site-controlled Delivery System: A Study on Process Parameters and in vitro Performances." Eur. J. Pharm. Sci. 22 (5): 469-76.

[7] Sungthongjeen, S., Puttipipatkhachorn, S., Paeratakul, O.,
Dashevsky, A., and Bodmeier, R. 2004. "Development of Pulsatile Release Tablets with Swelling and Rupturable Layers." Journal of Control Release 95 (2): 147-59.

[8] Saettone, M. F., and Salminen, L. 1995. "Polymers Used in Ocular Dosage Form and Drug Delivery Systems." $A d v$. Drug Deliv. Rev. 16 (2): 95-106.

[9] Gupta, A., Tiwari, G., Tiwari, R., and Srivastava, R. 2015. "Factorial Designed 5-Fluorouracil-Loaded Microsponges and Calcium Pectinate Beads Plugged in Hydroxypropyl Methylcellulose Capsules for Colorectal Cancer." Int. J. Pharm. Invest. 5 (4): 234-46.

[10] Varshosaz, J., Tavakoli, N., and Eram, S. A. 2006. "Use of Natural Gums and Cellulose Derivatives in Production of Sustained Release Metoprolol Tablets." Drug Deliv. 13 (2): 113-9.

[11] Patiwala, M. S. M., Jethara, S. I., Mukesh, R., and Patel, M. R. 2015. "Optimizing Oral Controlled Release Drug Delivery Systems Using Experimental Designs.” World J. Pharm. Res. 10.4712: 2745-56.

[12] Callewaert, M., Millot, J., Lesage, J., Laurent-Maquin, D., and Edwards-Levy, F. 2009. "Serum Albumin-Alginate Coated Microspheres: Role of the Inner Gel in Binding and Release of the KRFK Peptide." Intl. J. Pharm. 366 (1-2): 103-10.

[13] Ummadi, S. B., Shravani, N. G., Raghavendra, R. M., Srikanth, R., and Sanjeev, N. B. 2013. "Overview of Controlled Release Dosage Form.” Int. J. Pharma. Sci. 3 (4): 258-69.

[14] Agrawal, P. 2014. "Significance of Polymers in Drug Delivery System.” J. Pharmacovigi 3 (1): 1-2. 